\title{
Simple Statistical Analysis of the Impact of Some Nonidealities in Downstream VDSL with Linear Precoding
}

\author{
Marco Baldi, ${ }^{1}$ Franco Chiaraluce, ${ }^{1}$ Roberto Garello, ${ }^{2}$ Marco Polano, ${ }^{3}$ and Marcello Valentini ${ }^{3}$ \\ ${ }^{1}$ Dipartimento di Ingegneria Biomedica, Elettronica e Telecomunicazioni, Università Politecnica delle Marche, 60131 Ancona, Italy \\ ${ }^{2}$ Dipartimento di Elettronica, Politecnico di Torino, 10129 Torino, Italy \\ ${ }^{3}$ Telecom Italia, Via Guglielmo Reiss Romoli 274, 10148 Torino, Italy
}

Correspondence should be addressed to Franco Chiaraluce, f.chiaraluce@univpm.it

Received 1 June 2010; Revised 27 August 2010; Accepted 16 September 2010

Academic Editor: George Tombras

Copyright (C) 2010 Marco Baldi et al. This is an open access article distributed under the Creative Commons Attribution License, which permits unrestricted use, distribution, and reproduction in any medium, provided the original work is properly cited.

\begin{abstract}
This paper considers a VDSL downstream system where crosstalk is compensated by linear precoding. Starting from a recently introduced mathematical model for FEXT channels, simple analytical methods are derived for evaluating the average bit rates achievable, taking into account three of the most important nonidealities. First, absolute and relative estimation errors in the crosstalk coefficients are discussed, and explicit formulas are obtained to express their impact. A simple approach is presented for computing the maximum line length where linear precoding overcomes the noncoordinate system. Then, the effect of out-ofdomain crosstalk is analyzed. Finally, quantization errors in precoding coefficients are considered. We show that by the assumption of a midtread quantization law with different thresholds, a relatively small number of quantization bits is sufficient, thus reducing the implementation complexity. The presented formulas allow to quantify the impact of practical impairments and give a useful tool to design engineers and service providers to have a first estimation of the performance achievable in a specified scenario.
\end{abstract}

\section{Introduction}

As well known, the performance of very high speed digital subscriber line (VDSL) systems is basically limited by crosstalk. Generally, near-end crosstalk (NEXT) is not a problem, since it can be easily avoided by using frequency division duplexing. Far-end crosstalk (FEXT) is much more critical. It can be of in-domain type, when all the lines of a binder are controlled by a single operator and terminate on the same line card, or of out-of-domain (alien) type, when more operators provide services within the same binder (or a single operator is not able to guarantee that all the binder lines terminate on the same line card).

Several processing techniques have been proposed to eliminate the FEXT. Focusing on the downstream transmissions, that will be considered in the following, a very efficient solution consists in using a Diagonalizing Precoder (DP) [1]. It is based on a channel diagonalizing criterion and has a much lower complexity than competing solutions, like the Tomlinson-Harashima Precoder (THP) [2], since it does not require any additional receiver-side operation. (In order to further reduce complexity, a simplified version of DP has also been proposed in [3].)

The main concern of the DP approach is that precise estimates of the crosstalk channels are needed; these are usually found by using multiple-input multiple-output (MIMO) channel identification techniques, with some information communicated back to the transmitter side. Classic estimation techniques, like Least Mean Squares (LMS) and its variants [4-6], can be employed. (Algorithms for fast estimation have also been presented in [7-9].) Unfortunately, errors occurring in the estimation can reduce the achievable capacity, particularly for short line lengths. As we will show in this paper, LMS is indeed able to guarantee very small errors and, hence, effective precompensation. Once the crosstalk channels have been determined, however, they cannot be retained valid for all time: temperature changes and lines activation/deactivation oblige to update the estimation [10]; in other words, the precoder must track variations in the crosstalk environment [11].

Moving from these premises, a valuable task consists in evaluating the impact of FEXT estimation on the VDSL 
system capacity, in terms of both absolute errors (due to the estimation algorithm) and relative errors (induced by the crosstalk channel variations). Typically, this kind of problems is faced through measurements, by invoking the specificities of each implementation [12]. However, simple analytical expressions would be very useful for design engineers to have a first idea of the achievable performance and correctly address the design without resorting to long measurement campaigns.

Previous literature is rather poor of contributions of this kind. Among the most significant papers in the field, a statistical analysis has been outlined in [13], where the authors, however, do not refer to any practical model and do not elaborate on the analytical problem. Very recently, in [14], random variable theory has been applied in the context of dynamic spectrum management algorithms at level 2 (i.e., without distortion compensation). A two-port FEXT estimator proposed by the same authors was considered, and a statistical sensitivity analysis was conducted to investigate the effects on the system capacity of measurement errors due to uniform quantization.

Indeed, the problem of calculating the effect of estimation errors is made involved by the need of a reliable analytical model for the crosstalk channels. Until now, DSL standards usually relied on the so-called $1 \%$ worst-case model [15], which means that there is only a $1 \%$ chance that the actual FEXT coupling strength in a real bundle is worse than some value prefixed by the standard. Actually, the inappropriateness of the $1 \%$ worst-case model, particularly when applied to complex scenarios (i.e., with different interferers), has been widely debated [16], and an improved Full Service Access Network (FSAN) method has also been accepted as a standard [17]. More recently, two relevant contributions on FEXT modelling have been produced [18, 19]. Both, they describe the FEXT coupling dispersion by using a Gaussian variable or a Beta distribution, respectively, to model the amplitude, and a uniform distribution to model the phase. (In [19], the phase exhibits an additional contribution due to the direct channel.) As it will be shown afterwards, by exploiting such new models, the effect of the estimation errors can be described in statistical terms by obtaining, for example, the mean value of the bit loading in nonideal conditions.

The Gaussian channel model in [18] well matches European cables, while the Beta channel model of [19] is more tailored for North American cables. As we are mainly interested in considering European settings, the analytical treatment developed in this paper focuses on the Gaussian channel model. Its main statistical features will be derived in Section 2.3.

The object of this paper is to start from the FEXT channel model and to formulate a simple analytical framework for the calculation of the average bit rates in the presence of estimation errors, by taking into account the stochastic nature of the channel model. A relevant feature of the proposed analysis is that it can also be applied to the outof-domain crosstalk, this way permitting to evaluate the impact of such a further interference contribution, without the need of long simulations or measurements. Moreover, as the precoding system is also affected by quantization errors, we can evaluate in the same way the effect of finite word length in the representation of precoder variables. This issue has been faced only recently in the literature [20], but it is extremely important due to its influence on the performance/complexity tradeoff: coarse quantization can imply an intolerable loss but, on the other hand, a large number of quantization bits can yield high hardware complexity and a great amount of memory needed for the precoding process. In [20], it has been shown that to obtain a capacity loss, due to quantization errors, below a prefixed small percentage, a 14 bits representation of the precoder entries is necessary. We will verify that by adopting a quantization law that exploits the row-wise diagonal dominant (RWDD) character of the downstream VDSL channel, the same loss can be reached by adopting a smaller number of bits.

The organization of the paper is as follows. In Section 2, we remind the structure of the considered precoding system. In Section 3 we face the problem of residual absolute estimation errors, and we also write conditions that permit to establish the superiority, on average, of the vectored system against the nonvectored system. In Section 4, for the case of relative errors, we consider three different approximations of the average bit rate; the effect of uncertainty in the knowledge of the channel statistical parameters is discussed as well. In Section 5, the analysis is extended to the outof-domain (alien) crosstalk, by evaluating its impact in absence of cancellation techniques. In Section 6, the same statistical approach is adopted to estimate the rate loss due to quantization errors in representing the elements of the precoding matrix, by using different quantization laws and different numbers of quantization bits. The validity of the theoretical analysis presented in Sections 2-6 is confirmed by several numerical examples at the end of each section. Conclusions are drawn in Section 7.

\section{System Description}

In this paper, we consider the VDSL 99817 standard [21], characterized by 4096 tones with frequency separation $\Delta=4312.5 \mathrm{~Hz}$, focusing attention on the downstream transmission. Noting by $s_{k}^{\text {mask }}$ the value fixed by the standard [21] for the Power Spectral Density (PSD) at the $k$ th tone, the power transmitted on line $n$ at tone $k$ must satisfy the constraint $P_{k}^{n} \leq s_{k}^{\text {mask }} \Delta$. On each line, we consider a total power $P_{T}^{n}=\sum_{k} P_{k}^{n}$ equal to $14.5 \mathrm{dBm}$ (a typical value for cabinet transmission), distributed by the water-filling algorithm (see, e.g., [22]) on the 2454 tones allocated for downstream.

The scheme of Figure 1 refers to $L$ lines in the same binder. In the figure:

(i) $\mathbf{X}_{k}=\left[\mathrm{X}_{k}^{1}, X_{k}^{2}, \ldots, X_{k}^{L}\right]^{T}$ is an $L$-component vector grouping the symbols transmitted on tone $k$ by each of the $L$ users;

(ii) $\mathbf{H}_{k}=\left\{H_{k}^{i j}\right\}$ is the $L \times L$ channel matrix: the diagonal terms $H_{k}^{i i}$ represent the direct channels, while the other terms $H_{k}^{i j}, i \neq j$, represent the FEXT; 


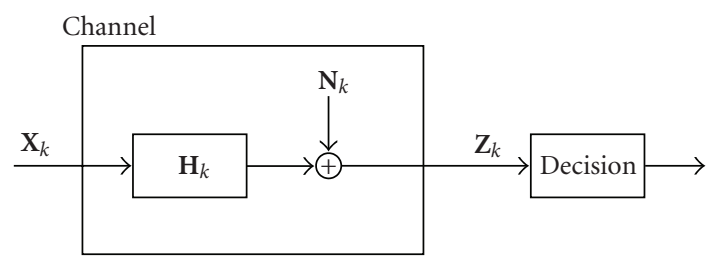

FIgURE 1: VDSL channel for $L$ lines in a binder.

(iii) $\mathbf{N}_{k}$ is an $L$-component vector describing the additive thermal noise contributions $N_{k}^{i}$.

The matrix $\mathbf{H}_{k}$ is RWDD; this means that, on each row of $\mathbf{H}_{k}$, the diagonal element has typically much larger magnitude than the off-diagonal elements (i.e., $\left|H_{k}^{i i}\right| \gg$ $\left|H_{k}^{i j}\right|$, for all $\left.j \neq i\right)$. Such RWDD character will be verified numerically in Section 2.4.

The signal-to-noise ratio for the $n$th receiver at the $k$ th tone, in the presence of FEXT, is

$$
\widehat{\operatorname{SNR}}_{k}^{n}=\frac{P_{k}^{n}\left|H_{k}^{n n}\right|^{2}}{\sum_{j \neq n}\left|H_{k}^{n j}\right|^{2} P_{k}^{j}+\sigma_{N}^{2}},
$$

where $\sigma_{N}^{2}$ is the variance of the thermal noise (independent of $k$ and $n$ ): a constant noise power spectral density equal to $-140 \mathrm{dBm} / \mathrm{Hz}$ will be considered in the numerical examples throughout the paper.

By using the well-known gap approximation, the number of bits/symbol of user $n$ at tone $k$ is given by

$$
c_{k}^{n}=\left[\min \left\{\log _{2}\left(1+\frac{\widehat{\mathrm{SNR}}_{k}^{n}}{\Gamma}\right), c_{\max }\right\}\right],
$$

where $[z]$ is the integer part of $z, \Gamma$ is the transmission gap, and $c_{\max }$ represents the maximum admitted value for the number of bits on each tone for VDSL (bit clipping).

The value of $\Gamma$ includes the nonideality of QAM constellation at a given bit error rate, the coding gain and the system margin. In this paper, we will assume a value $\Gamma=12.8 \mathrm{~dB}$, that is typical for practical implementations [13]. Moreover, according to the VDSL standard [21], we will consider $c_{\max }=$ 15 bits (the largest constellation allowed is a 32768-QAM). by

The achievable bit rate, expressed in bit/s, is then given

$$
C^{n}=R_{S} \sum_{k=1}^{Q} c_{k}^{n}
$$

where $R_{S}=4000 \mathrm{symbol} / \mathrm{s}$ is the net symbol rate (which differs from $\Delta$ because of the cyclic prefix), and $Q$ is the number of tones available for each user.

2.1. Diagonalizing Precoder. If all the $L$ lines of the binder are controlled by the same operator, and the line drivers are colocated (in the same cabinet or central office), then the vector of symbols $\mathbf{X}_{k}$ can be made available to an apparatus able to coordinate the $L$ lines. Ideally, this knowledge can

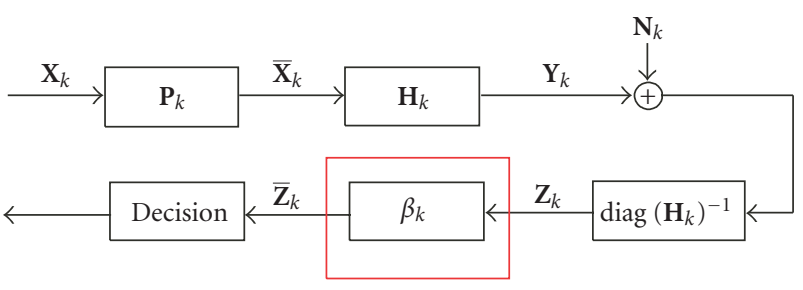

FIGURE 2: Schematic representation of the vectored system based on DP.

be used to completely eliminate the FEXT interference by applying a proper precoder [2].

In ideal conditions, that is, when all the channel elements $H_{k}^{i j}$ are perfectly known, the FEXT is removed and the signalto-noise ratio for the $n$th receiver at the $k$ th tone is

$$
\mathrm{SNR}_{k}^{n}=\frac{P_{k}^{n}\left|H_{k}^{n n}\right|^{2}}{\sigma_{N}^{2}}
$$

that, inserted in (2) (in place of $\widehat{\mathrm{SNR}}_{k}^{n}$ ) and (3), provides the achievable bit rates: they can be considerably larger than those of the noncoordinate system.

Among the solutions proposed in the literature to realize precoding, the so called Diagonalizing Precoder (DP) [1] is particularly effective. The DP system is schematically shown in Figure 2, with reference to the $k$ th downstream tone $f_{k}$. The diagonalizing precoder matrix $\mathbf{P}_{k}$ is defined as

$$
\mathbf{P}_{k}=\beta_{k}^{-1} \mathbf{H}_{k}^{-1} \operatorname{diag}\left(\mathbf{H}_{k}\right)
$$

with $\beta_{k} \triangleq \max _{i}\left\|\left[\mathbf{H}_{k}^{-1} \operatorname{diag}\left(\mathbf{H}_{k}\right)\right]_{\text {row } i}\right\|$.

It is possible to verify that, because of the RWDD character of the channel matrix, $\beta_{k}$ is always close to unity [1].

2.2. Channel Models. Equation (1) can be, obviously, applied in an experimental framework, where the values of $H_{k}^{n j}$ are determined by measurements. However, useful information can be obtained by developing a theoretical framework that aims at expressing the signal-to-noise ratio in simple analytical terms. For this purpose, a reliable channel model is required.

As regards the direct channel, a general consensus exists on the adoption of the so-called Marconi (MAR) model, which provides the value of $H_{k}^{n n}$ as a function of the frequency $f_{k}=k \Delta$ and the line length $d$ [23].

As for the crosstalk terms, in this paper, we adopt the model proposed in [18]. The starting point of the model is a multiple-input multiple-output (MIMO) extension of the MAR model, according to which the FEXT transfer function at frequency $f_{k}$ (in $\mathrm{MHz}$ ) from line $j$, of length $d_{j}$ (in $\mathrm{km}$ ), to line $n$, of length $d_{n}$, can be expressed as

$$
H_{k}^{n j}=\left|H_{k}^{n n}\right| f_{k} \sqrt{\min \left(d_{j}, d_{n}\right)} \chi 10^{-X / 20} e^{j \phi},
$$


where $\chi=10^{-2.25}$ is a coupling coefficient, and $X$ and $\phi$ are random variables. $X$ is described as a Gaussian variable, with mean value (in $\mathrm{dB}) \mu_{X}$ and standard deviation (in $\mathrm{dB}$ ) $\sigma_{X}$. The values of $\mu_{X}$ and $\sigma_{X}$ depend on the type of cable adopted but are related one each other as $\mu_{X}=2.33 \sigma_{X}$. As an example, in this paper, we consider the case of 10-pair binders for which $\mu_{X}=18.174 \mathrm{~dB}$ and $\sigma_{X}=7.8 \mathrm{~dB}$. The random variable $\phi$ is uniformly distributed between 0 and $2 \pi$.

This Gaussian model will be used for the subsequent analysis. As mentioned in the Introduction, recently a Beta channel model has also been proposed [19] that is more tailored for North American cables. The approach we present could be extended to cover the Beta model, too.

\subsection{Crosstalk Statistical Features for the Gaussian Channel} Model. The average value of $\left|H_{k}^{n j}\right|^{2}$ can be easily computed, and will be useful in the subsequent bit rate analysis. In fact, by (6), we can write

$$
\left|H_{k}^{n j}\right|^{2}=\left|H_{k}^{n n}\right|^{2} f_{k}^{2} \chi^{2} \min \left(d_{j}, d_{n}\right) 10^{-X / 10}
$$

As $X$ is a Gaussian variable, $Y=10^{-X / 10}$ is a log-normal variable whose mean value and variance are, respectively,

$$
\begin{gathered}
\langle Y\rangle=\mu_{Y}=\exp \left[-\frac{\ln 10}{10} \mu_{X}+\left(\frac{\ln 10}{10}\right)^{2} \frac{\sigma_{X}^{2}}{2}\right], \\
\sigma_{Y}^{2}=\left\{\exp \left[\left(\frac{\ln 10}{10}\right)^{2} \sigma_{X}^{2}\right]-1\right\} \exp \left[-2 \frac{\ln 10}{10} \mu_{X}+\left(\frac{\ln 10}{10}\right)^{2} \sigma_{X}^{2}\right] .
\end{gathered}
$$

So, as a consequence of (8), we can write

$$
\left\langle\left|H_{k}^{n j}\right|^{2}\right\rangle=\left|H_{k}^{n n}\right|^{2} f_{k}^{2} \chi^{2} \min \left(d_{j}, d_{n}\right) \mu_{Y}
$$

For the subsequent analysis, it will also be useful to know the statistical properties of

$$
I=\sum_{\substack{j=1 \\ j \neq n}}^{L} A_{j} \cdot 10^{-X_{j} / 10},
$$

where $X_{j}$ is a Gaussian variable and $A_{j}=\min \left(d_{j}, d_{n}\right) P_{k}^{j}$; thus, $I$ is the sum of $L-1$ properly weighted log-normal variables. It is generally well accepted that the distribution of I can be approximated by another log-normal distribution [24]. The mean value and the standard deviation of $I$ can be determined by using the so-called Wilkinson's method [25] that has the advantage to permit a simple and explicit analytical formulation. Other approaches are possible (like the Schwartz and Yeh's method [26]) and are even more accurate, but they require a recursive solution that does not allow for further analytical derivations.

By using Wilkinson's method, assuming that all $X_{j}$ 's have the same statistics and are uncorrelated one each other, it is

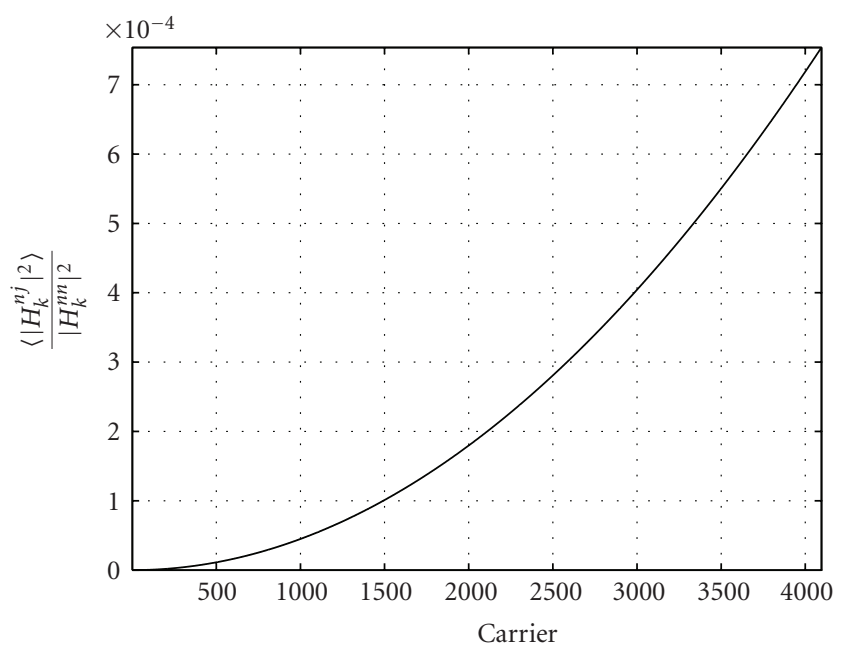

FIgURE 3: Average value of $\left|H_{k}^{n j}\right|^{2}$, normalized to the square modulus of the direct channel, for interfering lines of $1 \mathrm{~km}$.

easy to find

$$
\begin{gathered}
\langle I\rangle=\mu_{I}=\mu_{Y} \sum_{\substack{j=1 \\
j \neq n}}^{L} A_{j}, \\
\sigma_{I}^{2}=\sigma_{Y}^{2} \sum_{\substack{j=1 \\
j \neq n}}^{L} A_{j}^{2},
\end{gathered}
$$

this way generalizing (8) and (9).

It must be said that Wilkinson's method permits us to deal also with correlated $X_{j}$ 's; in such case, (12) still holds, while (13) should be modified for including the effect of the nonnull correlation coefficient [25]. In this paper, however, we only consider uncorrelated variables.

\subsection{Numerical Results: Verification of the RWDD Character for} the Channel Matrix. By using (8) and (10) and computing $\left|H_{k}^{n n}\right|^{2}$ through the MAR model, the ratio $\left\langle\left|H_{k}^{n j}\right|^{2}\right\rangle /\left|H_{k}^{n n}\right|^{2}$ can be determined, for a specific scenario. An example is shown in Figure 3, for the case $d_{j}=d_{n}=1 \mathrm{~km}$, as a function of the carrier frequency. This example confirms the RWDD character of the channel matrix.

\section{Effect of In-Domain Crosstalk Estimation Errors: Absolute Errors}

Let use denote by $\hat{\mathbf{H}}_{k}$ the estimated channel matrix at the $k$ th tone. If an estimation error is present, it can be modeled through a matrix $\mathbf{E}_{k}$ such that:

$$
\hat{\mathbf{H}}_{k}=\mathbf{H}_{k}+\mathbf{E}_{k} \text {. }
$$


Matrix $\hat{\mathbf{H}}_{k}$ should replace, in (5), the actual matrix $\mathbf{H}_{k}$. Looking at Figure 2 and by applying some algebra, we can compute the received symbol, which is given by

$$
\begin{aligned}
\overline{\mathbf{Z}}_{k}= & \left\{\mathbf{I}-\left[\operatorname{diag}\left(\hat{\mathbf{H}}_{k}\right)\right]^{-1} \cdot \operatorname{diag}\left(\mathbf{E}_{k} \cdot \hat{\mathbf{H}}_{k}^{-1}\right) \cdot \operatorname{diag}\left(\hat{\mathbf{H}}_{k}\right)\right\} \cdot \mathbf{X}_{k} \\
& -\left[\operatorname{diag}\left(\hat{\mathbf{H}}_{k}\right)\right]^{-1} \cdot\left[\mathbf{E}_{k} \cdot \hat{\mathbf{H}}_{k}^{-1}-\operatorname{diag}\left(\mathbf{E}_{k} \cdot \hat{\mathbf{H}}_{k}^{-1}\right)\right] \\
& \cdot \operatorname{diag}\left(\hat{\mathbf{H}}_{k}\right) \cdot \mathbf{X}_{k}+\beta_{k}\left[\operatorname{diag}\left(\hat{\mathbf{H}}_{k}\right)\right]^{-1} \cdot \mathbf{N}_{k},
\end{aligned}
$$

where I is the identity matrix.

3.1. Some Consequences of the RWDD Nature of the Channel Matrix. Since it is reasonable to assume that the direct channels are estimated correctly [2], $\mathbf{E}_{k}$ can be written as

$$
\mathbf{E}_{k}=\left[\begin{array}{cccc}
0 & \epsilon_{k}^{12} & \cdots & \epsilon_{k}^{1 L} \\
\epsilon_{k}^{21} & 0 & \cdots & \epsilon_{k}^{2 L} \\
\vdots & \vdots & \ddots & \vdots \\
\epsilon_{k}^{L 1} & \epsilon_{k}^{L 2} & \cdots & 0
\end{array}\right]
$$

As mentioned in Section 2.1, we can assume, $\beta_{k} \approx 1$. Moreover, in Appendix A, it is demonstrated that, because of the RWDD character of the channel matrix, $\operatorname{diag}\left(\mathbf{E}_{k} \cdot \hat{\mathbf{H}}_{k}^{-1}\right) \approx$ 0 .

By introducing these approximations, (15) can be simplified as follows:

$$
\overline{\mathbf{Z}}_{k} \approx \mathbf{X}_{k}-\left[\operatorname{diag}\left(\hat{\mathbf{H}}_{k}\right)\right]^{-1} \cdot \mathbf{E}_{k} \cdot \mathbf{X}_{k}+\left[\operatorname{diag}\left(\hat{\mathbf{H}}_{k}\right)\right]^{-1} \cdot \mathbf{N}_{k} .
$$

We note that the residual crosstalk due to the estimation error adds to the thermal noise contribution: a reduction in the achievable bit rate is therefore expected.

3.2. Absolute Errors for LS Methods. By assuming the adoption of a Least Square (LS) estimator [27], denoting by $S$ the length of the training sequence, the mean square value of the absolute error $\bar{\epsilon}_{k}^{n j}(S)$ on the estimation of $H_{k}^{n j}$ results in

$$
\left\langle\left|\bar{\epsilon}_{k}^{n j}(S)\right|^{2}\right\rangle=\frac{1}{S} \frac{\sigma_{N}^{2}}{P_{k}^{j}}
$$

This expression holds when the $H_{k}^{n j}$,s are individually estimated. In practical applications, a more efficient approach can be adopted, that consists in estimating simultaneously all the crosstalk coefficients, at the $k$ th tone, for the $n$th line. In this case, during the training phase, for a given frequency, all lines must transmit the same power, that is, it should be $P_{k}^{j}=$ $P_{k}$. Under such condition, we demonstrate in Appendix B that (18) is valid also in this case.

3.3. The Signal-to-Noise Ratio Expression Taking into Account Absolute Errors. Multiplying (18) by the power of the $j$ th transmitted signal and summing up the crosstalk contributions from $L-1$ interfering lines, the signal-to-noise ratio for the $n$th user at the $k$ th tone results in

$$
\mathrm{SNR}_{k}^{n}=\frac{P_{k}^{n}\left|H_{k}^{n n}\right|^{2}}{\sum_{j \neq n}\left\langle\left|\bar{\epsilon}_{k}^{n j}(S)\right|^{2}\right\rangle P_{k}^{j}+\sigma_{N}^{2}}=\frac{P_{k}^{n}\left|H_{k}^{n n}\right|^{2}}{((L-1) / S+1) \sigma_{N}^{2}} .
$$

Based on this very simple expression, in comparison with (4), we can say that the final effect of the absolute estimation error is to amplify the thermal noise by a factor $[1+(L-1) / S]$. So, if the value of $S$ is sufficiently large, the impact of the estimation error after application of the LS procedure can be made negligible. This will be shown next through numerical examples.

3.4. Estimation of the Maximum Line Length where the DP Improves the System. The previous analysis allows to estimate the line length above which, if the channel is measured by the LS method, the DP loses its advantage with respect to the noncoordinate system. By comparing (19) with (1), that refers to the case without precoding, we can derive the condition by which vectoring provides, on average, a greater signal-to-noise ratio on the $n$th line and the $k$ th tone, and then, a greater (or, at least, equal) bit rate. This occurs as long as the following inequality is satisfied

$$
\sum_{j \neq n}\left|H_{k}^{n j}\right|^{2} P_{k}^{j} \geq \frac{L-1}{S} \sigma_{N}^{2}
$$

This condition can be extended to the whole set of downstream tones for the $n$th line

$$
\sum_{k} \sum_{j \neq n}\left|H_{k}^{n j}\right|^{2} P_{k}^{j} \geq Q \frac{L-1}{S} \sigma_{N}^{2},
$$

and to the whole set of active lines

$$
\sum_{n} \sum_{k} \sum_{j \neq n}\left|H_{k}^{n j}\right|^{2} P_{k}^{j} \geq L \cdot Q \frac{L-1}{S} \sigma_{N}^{2} .
$$

As in (20)-(22), even taking into account its statistical nature, the modulus of $H_{k}^{n j}$ decreases for increasing lengths, a threshold length should exist above which the application of DP is no longer expedient.

More precisely, although (20)-(22) can be applied in specific scenarios, and then for specific values of $H_{k}^{n j}$, it can be useful, for a design engineer or a service provider, to have an idea of the maximum lengths achievable by considering the average crosstalk power. Such information can be obtained by replacing $\left|H_{\mathrm{k}}^{n j}\right|^{2}$ with $\left\langle\left|H_{k}^{n j}\right|^{2}\right\rangle$. So, by using (12), with $A_{j}=\min \left(d_{j}, d_{n}\right) P_{k}^{j}$, condition (20) becomes

$$
\begin{gathered}
\left|H_{k}^{n n}\left(d_{n}\right)\right|^{2} f_{k}^{2} \chi^{2} \exp \left[-\frac{\ln 10}{10} \mu_{X}+\left(\frac{\ln 10}{10}\right)^{2} \frac{\sigma_{X}^{2}}{2}\right] \\
\cdot \sum_{j \neq n} \min \left(d_{j}, d_{n}\right) P_{k}^{j} \geq \frac{L-1}{S} \sigma_{N}^{2},
\end{gathered}
$$




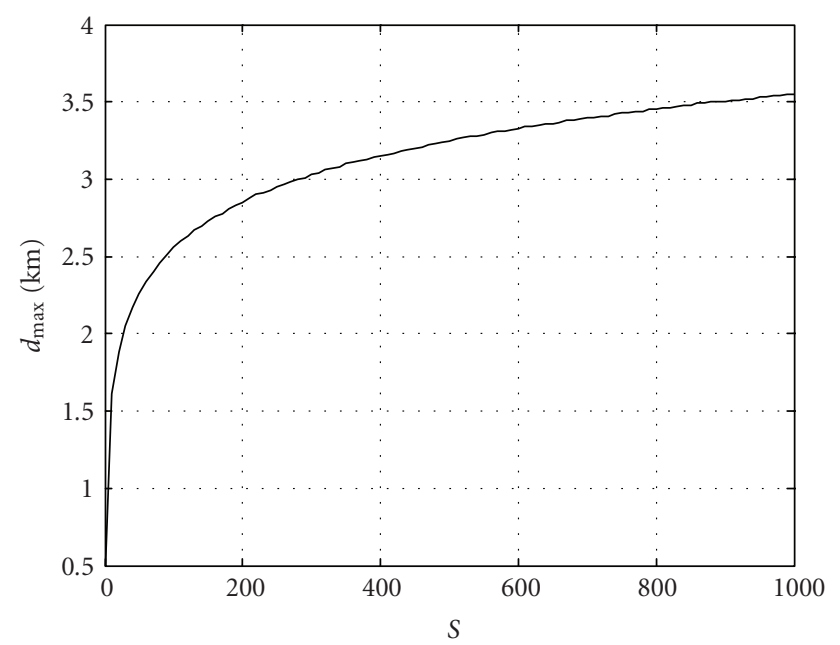

Figure 4: Maximum value of $d$, in a system with lines of equal length, for which DP outperforms the nonvectored scheme, as a function of the number of training symbols $S$.

where the dependence of $H_{k}^{n n}$ on the line length has also been written for clarity. The same substitution can be done in (21) and (22).

3.5. Numerical Results: Performance in the Presence of Absolute Estimation Errors. Let us consider a scenario with $L=8$ and four different line lengths $d_{i}, i=1, \ldots, 8: d_{1}=d_{2}=0.3 \mathrm{~km}$, $d_{3}=d_{4}=0.6 \mathrm{~km}, d_{5}=d_{6}=0.9 \mathrm{~km}, d_{7}=d_{8}=1.2 \mathrm{~km}$. The average bit rates, as functions of the number of training symbols, are shown in Table 1, and compared with the results of the nonvectored scheme (obtained through simulationsee Table 2) and the ideal vectored scheme. From the table, we see that, just by using $S=100$ training symbols, the average bit rate is very close to the ideal result, thus providing the expected gain with respect to the nonvectored system.

As an example of application of the formulas in Section 3.4, let us consider a scenario with lines of equal length $d$. We wish to find the maximum length, denoted by $d_{\max }$, above which application of vectoring is no longer useful. The cost function adopted is the overall bit rate for each user, which implies to study condition (21). Under the established assumptions, the average of (23) over the $Q$ tones results in

$$
2.42 \cdot 10^{-6} d \sum_{k=1}^{Q}\left|H_{k}(d)\right|^{2} f_{k}^{2} P_{k} \geq Q \frac{\sigma_{N}^{2}}{S}
$$

as $H_{k}^{n n}$ does not depend on $n$ and $P_{k}^{j}$ does not depend on $j$. It is also interesting to observe that this expression is independent of the number of lines. This is a consequence of the fact that we are analyzing the average behavior. The plot of $d_{\max }$, as a function of $S$, is reported in Figure 4 . The figure shows that just assuming $S$ in the order of 100 , vectoring is convenient for any line length of practical interest (i.e., $<2.5 \mathrm{~km}$ ). Obviously, this favorable conclusion implies the implementation of an ideal LS estimator, that is able to ensure the mean square value of the estimation error given by (18).

\section{Effect of In-Domain Crosstalk Estimation Errors: Relative Errors}

The analysis developed in the previous section demonstrates that, by using an effective estimation algorithm, the residual estimation errors have not a significant impact on the bit loading achievable. The previous analysis, however, relies on two important assumptions:

(i) there is no quantization noise in representing the matrix coefficients at the precoder;

(ii) the crosstalk channels are static.

The impact of the quantization noise will be discussed in Section 6. In this section, instead, we study in statistical terms, that is, by evaluating the average degradation, the effect of a change in the crosstalk contributions after the precoder has been synchronized.

The crosstalk environment can vary, for example, as a consequence of a temperature change or lines activation/deactivation. To cope with these variations, adaptive training algorithms can be adopted [28]. Adaptive algorithms require almost continuous transmission of information about the error at the output of the frequency-domain equalizer (FEQ) at the receiver; such information flows from the VDSL2 Transceiver Unit at the remote side (VTU-R) to the vectoring control entity (VCE) at the Digital Subscriber Line Access Multiplexer (DSLAM). This transmission can be a critical issue, as only a very low data rate special operations channel may be available to feed back the error samples. On the other hand, precoder updating should be fast.

Although clever solutions can be conceived for overcoming the problem of low data rate over the upstream channel [11], to evaluate the impact of modified crosstalk conditions remains a valuable task. As mentioned in the Introduction, the topic has been faced in the past by considering worstcase conditions or simplified statistical approaches. Next, we demonstrate that it is possible to find explicit formulas that permit to estimate the degradation in the achievable bit rate under more realistic assumptions.

4.1. The Signal-to-Noise Ratio Expression Taking into Account Relative Errors. Let us assume that, because of a channel change, the crosstalk coefficients are known, at the precoder, with a relative (percent) error $e$. (For the sake of simplicity, we assume that the relative error is the same for all coefficients; the analysis could be easily extended by removing such hypothesis.) This means that the error matrix $\mathbf{E}_{k}$ can be written as:

$$
\mathbf{E}_{k}=e\left[\begin{array}{cccc}
0 & H_{k}^{12} & \cdots & H_{k}^{1 L} \\
H_{k}^{21} & 0 & \cdots & H_{k}^{2 L} \\
\vdots & \vdots & \ddots & \vdots \\
H_{k}^{L 1} & H_{k}^{L 2} & \cdots & 0
\end{array}\right]
$$


TABLE 1: Example of average bit rates as functions of the number of training symbols.

\begin{tabular}{lcccccc}
\hline $\begin{array}{l}\text { Line length } \\
(\mathrm{km})\end{array}$ & $\begin{array}{c}\text { Nonvectored } \\
(\mathrm{Mbps})\end{array}$ & $\begin{array}{c}\text { Vectored } S=1 \\
(\mathrm{Mbps})\end{array}$ & $\begin{array}{c}\text { Vectored } S=10 \\
(\mathrm{Mbps})\end{array}$ & $\begin{array}{c}\text { Vectored } S=100 \\
(\mathrm{Mbps})\end{array}$ & $\begin{array}{c}\text { Vectored } S=1000 \\
(\mathrm{Mbps})\end{array}$ & $\begin{array}{c}\text { Vectored ideal } \\
(\mathrm{Mbps})\end{array}$ \\
\hline 0.3 & 88.50 & 127.81 & 138.54 & 140.98 & 141.22 & 141.25 \\
0.6 & 69.51 & 71.81 & 88.57 & 93.40 & 54.11 & 54.19 \\
0.9 & 49.36 & 42.76 & 54.49 & 37.98 & 37.42 & 37.65 \\
1.2 & 34.77 & 28.12 & 35.12 & & & 37.67 \\
\hline
\end{tabular}

Using expression (17) for the received symbol, the signalto-noise ratio for the $n$th user at the $k$ th tone, that takes into account the presence of the relative error $e$, is

$$
\operatorname{SNR}_{k}^{n}=\frac{P_{k}^{n}\left|H_{k}^{n n}\right|^{2}}{|e|^{2} \sum_{j \neq n}\left|H_{k}^{n j}\right|^{2} P_{k}^{j}+\sigma_{N}^{2}} .
$$

We observe that assuming $e=-1$ results in the nonvectored system; correspondingly, (26) reduces to (1).

4.2. Techniques for Estimating the Impact of Relative Errors. Let us define

$$
a=\frac{P_{k}^{n}\left|H_{k}^{n n}\right|^{2}}{\Gamma}, \quad b=|e|^{2}\left|H_{k}^{n n}\right|^{2} f_{k}^{2} \chi^{2},
$$

and let us take into account the definition of $I$, given by (11), whose mean value and variance have been computed in Section 2.3.
Wishing to find the average bit rate, taking into account the statistical features of $H_{k}^{n j}$ for a fixed value of $e$ (assumed as a parameter), a first possibility consists in replacing, in (26), the mean value of $\left|H_{k}^{n j}\right|^{2}$. This way, we find

$$
\left\langle c_{k}^{n}\right\rangle_{1}=\left[\min \left\{\log _{2}\left(1+\frac{a}{b \mu_{I}+\sigma_{N}^{2}}\right), c_{\max }\right\}\right]
$$

where $\mu_{I}$ is given by (12). We call this approach Approximation 1 .

A more accurate analysis consists in determining the probability density function (p.d.f.) of the $\mathrm{SNR}_{k}^{n}$ in (26), and then deriving the mean value of $c_{k}^{n}$ accordingly. In this case, it is easy to find

$$
\left\langle c_{k}^{n}\right\rangle_{2}=\left[\min \left\{\left\langle c_{k}^{n}\right\rangle_{1}+\log _{2}\left[\sqrt{\frac{\left(b \mu_{I}+\sigma_{N}^{2}\right)^{2}+b^{2} \sigma_{I}^{2}}{\left(b \mu_{I}+a+\sigma_{N}^{2}\right)^{2}+b^{2} \sigma_{I}^{2}}}\left(1+\frac{a}{b \mu_{I}+\sigma_{N}^{2}}\right)\right], c_{\max }\right\}\right],
$$

where $\sigma_{I}^{2}$ is given by (13), we call this approach Approximation 2.

Sometimes, to simplify the analysis (also in a simulator), another method can be used, which consists in neglecting $\sigma_{X}^{2}$ in (8). We call this approach Approximation 3 and denote the corresponding estimated average number of bits per symbol as $\left\langle c_{k}^{n}\right\rangle_{3}$. As, by this choice, the crosstalk power is underestimated, we expect that Approximation 3 provides too optimistic values for the expected bit rate.

For the sake of comparison, it can be useful to consider also the standard $1 \%$ worst-case model. The presence of different interferers, that is, characterized by different coupling lengths and transmit powers, is taken into account through the FSAN method [29]. Noting by $U$ the number of different interferer types and by $l_{i}$ the number of interferers of type $i$ (that is with length $d_{i}$ and transmit power $P_{k}^{i}$ ), the number of bits/symbol using the FSAN method results in

$$
\left(c_{k}^{n}\right)_{\mathrm{FSAN}}=\left[\min \left\{\log _{2}\left(1+\frac{a}{b\left(\sum_{i=1}^{U} A_{i}^{1 / 0.6} l_{i}\right)^{0.6}+\sigma_{N}^{2}}\right), c_{\max }\right\}\right]
$$

with $A_{i}=\min \left(d_{i}, d_{n}\right) P_{k}^{i}$; moreover, $b$ is computed from (27) assuming $|e|=1$.

Although the FSAN method certainly improves the way to sum crosstalk from different sources, the $1 \%$ worst-case model is not able to capture the positive effects of coupling dispersion. For this reason, it usually provides too pessimistic values for the expected bit rate.

Note that it may be interesting to extend the statistical analysis beyond the mere evaluation of the average values, for example to analyze the dispersion around the mean. In this case, the presented approach permits to derive, by simulation, the plots of the cumulative distribution function (c.d.f.), defined as the probability that the bit rate is equal 
to or smaller than a given value. In turn, by making the derivative of the c.d.f., the p.d.f. can be obtained.

The numerical results relative to the proposed approximations and the c.d.f. behavior will be presented in Section 4.4. In the next subsection, instead, we address another potential nonideality.

4.3. Uncertainty in the Knowledge of $\sigma_{X}$. The previous analysis assumes the knowledge of the standard deviation $\sigma_{X}$ (and, hence, the mean value $\mu_{X}$ ). Really, this parameter usually results from a campaign of measurements that obviously can suffer some uncertainty level. In particular, in our analysis for the case of 10-pair binders, we have used a set of data measurements provided by Telecom Italia. Based on these data, we have established that a $95 \%$ confidence interval is lower bounded by $\left.\sigma_{X}\right|_{\text {l.b. }}=7.4 \mathrm{~dB}$ and upper bounded by $\left.\sigma_{X}\right|_{\text {u.b. }}=8.1 \mathrm{~dB}$. Corresponding bounds can be found for the mean value $\mu_{X}$ as well, by using the relationship $\mu_{X}=$ $2.33 \sigma_{X}$, that are: $\left.\mu_{X}\right|_{\text {l.b. }}=17.242 \mathrm{~dB}$ and $\left.\mu_{X}\right|_{u . b .}=18.873 \mathrm{~dB}$. Once having defined the range, we have explored possibile sensitivity of the bit rates on such variability. Results are shown in the next subsection.

4.4. Numerical Results: Performance in the Presence of Relative Estimation Errors. Let us consider a scenario with $L=$ 8 and four different line lengths $d_{i}$, with $i=1, \ldots, 8$ : $d_{1}=d_{2}=0.3 \mathrm{~km}, d_{3}=d_{4}=0.6 \mathrm{~km}, d_{5}=d_{6}=$ $0.9 \mathrm{~km}, d_{7}=d_{8}=1.2 \mathrm{~km}$. Table 2 shows the estimated average bit rates $\left\langle C^{n}\right\rangle_{i}=R_{S} \sum_{k=1}^{Q}\left\langle c_{k}^{n}\right\rangle_{\mathrm{i}}, i=1,2,3$, for some values of $e$, according with the three approximations presented in Section 4.2. The case $e=-1$ corresponds to the nonvectored system. Actually, in all approximations, only the $|e|$ concurs to determine the estimated value. However, the sign of $e$ must be taken into account when deriving the expected bit rate through simulations. The latter consist of generating samples of the crosstalk coefficients, according with the specified statistics, without using the analytical expressions. So, they provide reference values the approximated results must compare with. Actually, in the table, the results of two different simulations are shown, the former using the exact expression (15) and the latter the simplified expression (17). The difference between these two approaches is almost negligible, as expected, being related with the RWDD character of matrix $\mathbf{H}_{k}$. From the table, we see that Approximation 2 generally gives results that are in good agreement with the simulation, particularly for the shortest lengths; Approximation 1 may underestimate the true values whilst, conversely, Approximation 3 may overestimate, even significantly, the true values. The last column in Table 2 shows the behavior of $\left\langle C^{n}\right\rangle_{\text {FSAN }}=$ $R_{S} \sum_{k=1}^{Q}\left(c_{k}^{n}\right)_{\mathrm{FSAN}}$. As expected, the values derived from the $1 \%$ worst-case method, that is at the basis of the FSAN approach, are smaller than those obtained from the statistical analysis.

As mentioned before, the statistical analysis can be integrated by the computation of the c.d.f. curves. Simulation is used for such purpose. The c.d.f.'s of the bit rates for $e=-0.5$ are plotted, by considering the above scenario, in Figure 5.

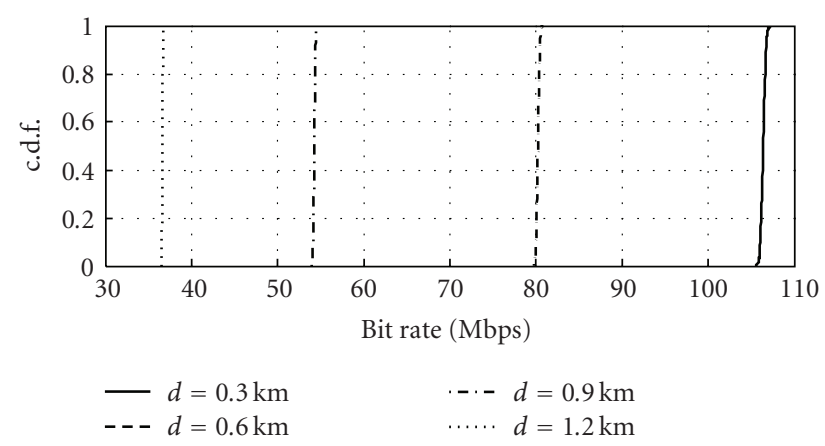

FIgURE 5: Estimated c.d.f. with $e=-0.5$.

We see that the dispersion around the mean, for all lengths, is very limited, so that the average value gives a very good approximation of the true value.

Finally, Table 3 shows the average bit rates for the nonvectored system $(e=-1)$, considering the mean value of $\sigma_{X}$ as well as the lower and the upper bounds on the $95 \%$ confidence interval. The ideal bit rate, achieved by perfect compensation of the crosstalk, is also reported as a reference. From the table we see that the sensitivity of the average bit rate on the parameters identifying the model is rather limited: the change in the precoding gain, for example, is in the order of $5 \%$ for the shortest lengths and $1 \%$ for the longest lengths, when passing from the lower bound to the upper bound of the confidence interval.

\section{Effect of out-of-Domain Crosstalk}

Let us suppose that the $L$ active lines are also disturbed by $M$ out-of-domain crosstalk contributions. This means that $M$ lines within the binder are not controlled by the operator that, therefore, cannot apply to them the coordinated vectoring action.

5.1. Out-of-Domain Crosstalk Model. Let us denote by $\mathbf{G}_{k}=$ $\left\{G_{k}^{i j}\right\}$ the $L \times M$ matrix collecting this kind of contributions, and by $\mathbf{A}_{k}=\left[A_{k}^{1}, A_{k}^{2}, \ldots, A_{k}^{M}\right]^{T}$ the $M$-component vector of the out-of-domain signals. It is reasonable to assume that the symbols $A_{k}^{i}$ 's have the same properties of the $X_{k}^{i}$ 's.

Under the same approximations used in (17), the expression of the received symbol becomes

$$
\begin{aligned}
& \overline{\mathbf{Z}}_{k} \approx \mathbf{X}_{k}-\left[\operatorname{diag}\left(\hat{\mathbf{H}}_{k}\right)\right]^{-1} \cdot \mathbf{E}_{k} \cdot \mathbf{X}_{k}+\left[\operatorname{diag}\left(\hat{\mathbf{H}}_{k}\right)\right]^{-1} \\
& \cdot \mathbf{G}_{k} \cdot \mathbf{A}_{k}+\left[\operatorname{diag}\left(\hat{\mathbf{H}}_{k}\right)\right]^{-1} \cdot \mathbf{N}_{k} .
\end{aligned}
$$

So, even in the case of perfect in-domain crosstalk compensation, the $n$th line is affected by a disturbance at the $k$ th tone

$$
V_{k}^{n}=\sum_{j=1}^{M} G_{k}^{n j} A_{k}^{j}+N_{k}^{n} .
$$


TABLE 2: Example of average bit rates in the presence of relative estimation error $e$.

\begin{tabular}{|c|c|c|c|c|c|c|}
\hline $\begin{array}{l}\text { Line length } \\
(\mathrm{km})\end{array}$ & $\begin{array}{l}\text { Simulation based } \\
\text { on (15) (Mbps) }\end{array}$ & $\begin{array}{c}\text { Simulation based } \\
\text { on (17) (Mbps) }\end{array}$ & $\begin{array}{c}\left\langle C^{n}\right\rangle_{1} \\
(\mathrm{Mbps})\end{array}$ & $\begin{array}{c}\left\langle C^{n}\right\rangle_{2} \\
(\mathrm{Mbps})\end{array}$ & $\begin{array}{c}\left\langle C^{n}\right\rangle_{3} \\
(\mathrm{Mbps})\end{array}$ & $\begin{array}{c}\left\langle C^{n}\right\rangle_{\mathrm{FSAN}} \\
(\mathrm{Mbps})\end{array}$ \\
\hline \multicolumn{7}{|c|}{$e=-0.1$} \\
\hline 0.3 & 135.60 & 135.63 & 129.84 & 135.96 & 138.68 & 113.83 \\
\hline 0.6 & 92.61 & 92.65 & 92.78 & 93.38 & 93.94 & 87.31 \\
\hline 0.9 & 58.16 & 58.17 & 58.18 & 58.23 & 58.40 & 56.73 \\
\hline 1.2 & 37.62 & 37.62 & 37.60 & 37.60 & 37.66 & 37.15 \\
\hline \multicolumn{7}{|c|}{$e=-0.5$} \\
\hline 0.3 & 106.39 & 106.39 & 96.33 & 105.55 & 114.89 & 71.33 \\
\hline 0.6 & 80.23 & 80.24 & 78.17 & 83.70 & 88.40 & 60.58 \\
\hline 0.9 & 54.27 & 54.27 & 53.33 & 55.65 & 57.08 & 44.62 \\
\hline 1.2 & 36.64 & 36.64 & 35.88 & 36.78 & 37.27 & 31.39 \\
\hline \multicolumn{7}{|c|}{$e=-1$} \\
\hline 0.3 & 88.50 & 88.50 & 77.74 & 87.54 & 98.09 & 52.82 \\
\hline 0.6 & 69.51 & 69.51 & 65.09 & 72.98 & 79.95 & 44.82 \\
\hline 0.9 & 49.36 & 49.36 & 47.33 & 51.12 & 54.06 & 35.40 \\
\hline 1.2 & 34.77 & 34.77 & 32.85 & 34.96 & 36.19 & 26.08 \\
\hline
\end{tabular}

TABLE 3: Effect of uncertainty in the knowledge of $\sigma_{X}$ for the nonvectored system.

\begin{tabular}{lcccc}
\hline $\begin{array}{l}\text { Line length } \\
(\mathrm{km})\end{array}$ & $\begin{array}{c}\text { Nonvectored } \\
\left(\sigma_{X}=7.4 \mathrm{~dB}\right) \\
(\mathrm{Mbps})\end{array}$ & $\begin{array}{c}\text { Nonvectored } \\
\left(\sigma_{X}=7.8 \mathrm{~dB}\right) \\
(\mathrm{Mbps})\end{array}$ & $\begin{array}{c}\text { Nonvectored } \\
\left(\sigma_{X}=8.1 \mathrm{~dB}\right) \\
(\mathrm{Mbps})\end{array}$ & $\begin{array}{c}\text { Ideal vectored } \\
(\mathrm{Mbps})\end{array}$ \\
\hline 0.3 & 86.72 & 88.50 & 89.80 & 70.28 \\
0.6 & 68.45 & 69.51 & 49.70 & 54.19 \\
0.9 & 48.84 & 49.36 & 34.90 & 37.67 \\
1.2 & 34.56 & 34.77 & & 37.67 \\
\hline
\end{tabular}

The correlation properties of this overall noise have been studied in depth [30]; for the purposes of this paper, however, it is enough to determine the power of the extranoise that, under the usual hypotheses, can be obtained as

$$
\left\langle\left|V_{k}^{n}\right|^{2}\right\rangle=\sum_{j=1}^{M}\left\langle\left|G_{k}^{n j}\right|^{2}\right\rangle A T_{k}^{j}+\sigma_{N}^{2},
$$

where $A T_{k}^{j}$ is the power transmitted, at the $k$ th tone, on the $j$ th out-of-domain line.

Including the out-of-domain crosstalk contribution in (19), we obtain the signal-to-noise ratio in the presence of an absolute estimation error and noncompensated alien crosstalk:

$$
\operatorname{SNR}_{k}^{n}=\frac{P_{k}^{n}\left|H_{k}^{n n}\right|^{2}}{\sum_{j=1}^{M}\left\langle\left|G_{k}^{n j}\right|^{2}\right\rangle A T_{k}^{j}+((L-1) / S+1) \sigma_{N}^{2}} .
$$

Similarly, we can combine the out-of-domain contributions with the relative estimation errors analysis; for example, using Approximation 1 and writing explicitly the various contributions, (28) becomes

$$
\left\langle c_{k}^{n}\right\rangle_{1}=\left[\min \left\{\log _{2}\left(1+\frac{P_{k}^{n}\left|H_{k}^{n n}\right|^{2}}{|e|^{2} \sum_{j=1, j \neq n}^{L}\left\langle\left|H_{k}^{n j}\right|^{2}\right\rangle P_{k}^{j}+\sum_{j=1}^{M}\left\langle\left|G_{k}^{n j}\right|^{2}\right\rangle A T_{k}^{j}+\sigma_{N}^{2}} \frac{1}{\Gamma}\right), c_{\max }\right\}\right] .
$$

To compute (34) or (35), modeling of the out-of-domain crosstalk channels is also required. In general, the same model used for the in-domain contributions can be adopted.
So, by using the Gaussian channel model, (10) can be applied by replacing $\left\langle\left|H_{k}^{n j}\right|^{2}\right\rangle$ with $\left\langle\left|G_{k}^{n j}\right|^{2}\right\rangle$; in this case, however, $d_{j}$ is the length of the $j$ th out-of-domain interfering 
line whereas $d_{n}$ is the length of the considered in-domain disturbed line.

To evaluate the impact of the out-of-domain crosstalk, we introduce the following two parameters:

$$
\begin{aligned}
T_{1}^{n} & =\frac{\left\langle C_{I}^{n}\right\rangle-\left\langle C_{V A}^{n}\right\rangle}{\left\langle C_{I}^{n}\right\rangle} \cdot 100, \\
T_{2}^{n} & =\frac{\left\langle C_{V A}^{n}\right\rangle-\left\langle C_{N A}^{n}\right\rangle}{\left\langle C_{V A}^{n}\right\rangle} \cdot 100,
\end{aligned}
$$

where, with reference to the $n$th line:

(i) $C_{I}^{n}=$ ideal bit rate,

(ii) $C_{V A}^{n}=$ bit rate of the vectored system with alien noise,

(iii) $C_{N A}^{n}=$ bit rate of the nonvectored system with alien noise.

$T_{1}^{n}$ is a measure of the loss due to the presence of the alien noise, also in the case of negligible estimation error (when the value of $S$ is large); $T_{2}^{n}$ is a measure of the loss due to the absence of vectoring when the alien noise is also present.

5.2. Numerical Results: Performance in the Presence of out-ofDomain Crosstalk. Let us consider a scenario with $L=M=$ 4 and $S=1000$. Both for the in-domain and the out-ofdomain lines, the line lengths are: $d_{1}=0.3 \mathrm{~km} ; d_{2}=0.6 \mathrm{~km}$; $d_{3}=0.9 \mathrm{~km} ; d_{4}=1.2 \mathrm{~km}$. Table 4 shows the values of the rates and the corresponding $T_{1}^{n}$ and $T_{2}^{n}$ parameters.

As shown in this example, the impact of the alien crosstalk can be significant, yielding a great reduction in the achievable bit rate, particularly for the shortest lengths. Consequently, the potential advantage of precoding can be compromised if the out-of-domain noise problem is not efficiently solved. Recently, new architectures have been proposed, that permit to cancel both in-domain and outof-domain crosstalk, at the expense of increased complexity [31]. To limit complexity, the new architectures use partial cancellation techniques to apply compensation only where it yields the maximum benefit.

\section{Effect of Quantization Errors}

In a real implementation, the elements of the precoding matrix are quantized. This yields a further nonideality, whose effects can be limited, with reasonable complexity, through the adoption of a suitable quantization rule.

6.1. Analytical Model for the Quantization Errors and Rate Loss. Let us suppose that matrix $\mathbf{P}_{k}$ is represented by a finite precision matrix $\hat{\mathbf{P}}_{k}$ such that

$$
\widehat{\mathbf{P}}_{k}=\mathbf{P}_{k}+\mathbf{D}_{k}
$$

where $\mathbf{D}_{k}$ expresses the quantization error. The latter, in turn, can be related to a matrix $\Delta_{k}$ as follows:

$$
\Delta_{k}=\mathbf{P}_{k}^{-1} \cdot \mathbf{D}_{k}
$$

In ideal conditions, that is assuming arbitrary precision, we have $\Delta_{k}=\mathbf{D}_{k}=\mathbf{0}$. Through simple algebra, the signal-tonoise ratio for the $n$th receiver at the $k$ th tone in the presence of the quantization error is given by the following expression, that was already derived in [20]

$$
\overline{S N R}_{k}^{n}=\frac{\left|H_{k}^{n n}\right|^{2}\left|1+\Delta_{k}^{n n}\right|^{2} P_{k}^{n}}{\left|H_{k}^{n n}\right|^{2} \sum_{j \neq n}\left|\Delta_{k}^{n j}\right|^{2} P_{k}^{j}+\sigma_{N}^{2}},
$$

being $\Delta_{k}^{n j}$ the $(n, j)$ th element of $\Delta_{k}$. Equation (39) can be used to replace the signal-to-noise ratio in (2), thus reducing the achievable bit rate with respect to the ideal conditions. By investigating the statistical properties of $c_{k}^{n}$, in the presence of quantization errors, it is possible to find the number of quantization bits needed to have a penalty smaller than a prefixed percentage. In this view, an indepth analytical work was done in [20], where a number of bounds were determined, and their reliability tested through simulations. In that paper, however, the elements of $\mathbf{D}_{k}$ were modeled as random variables uniformly distributed in the range $\left[-2^{-v}, 2^{-v}\right]$, where $v$ is the number of quantization bits adopted. No specific quantization law was considered, but it was shown that to obtain a small capacity loss, a 14 bits representation of the precoder entries is necessary. In the following, we will show that a smaller number of bits can be adopted, by using a quantization law that exploits the RWDD property of the channel matrix.

Noting by $\bar{c}_{k}^{n}$ the number of bits/symbol for the $n$th user at the $k$ th tone, in the presence of quantization error, and using definitions (2) and (3), the effect of quantization errors on the bit rate can be measured by the per cent rate loss, defined as

$$
\frac{\left\langle L^{n}\right\rangle}{\left\langle C^{n}\right\rangle} \cdot 100=\frac{\sum_{k=1}^{Q}\left\langle L_{k}^{n}\right\rangle}{\left\langle C^{n}\right\rangle} \cdot 100,
$$

where $L_{k}^{n}=c_{k}^{n}-\bar{c}_{k}^{n}=\log _{2}\left\{\left(1+\Gamma^{-1} \operatorname{SNR}_{k}^{n}\right) /\left(1+\Gamma^{-1} \overline{S N R}_{k}^{n}\right)\right\}$ is the transmission rate loss for the $n$th receiver at the $k$ th tone. In this expression, $S N R_{k}^{n}$ is given by (4).

Taking into account that the modulus of the diagonal elements of matrix $\mathbf{P}_{k}$ is close to 1 , a first choice consists of assuming a midtread quantization law between -1 and 1. However, because of the RWDD property of matrix $\mathbf{H}_{k}$, the off-diagonal elements are very small. So, following this quantization law, most of the off-diagonal elements become zeros after the quantization, particularly in the case of rather small $v$ and low frequencies. Explicitly, this means that the vectoring procedure is made ineffective by the quantization law, in such region. In spite of this, for small values of $v$, the error due to the midtread quantization law is, on average, smaller than that resulting from the assumption of a uniform error. For achieving a small rate loss, however, a large number of quantization bits may still be required. A typical value $v \geq 14$ bits, identified in [20], is confirmed by the numerical example reported in Section 6.2.

Anyway, the value of $v$ can be reduced by using a smarter quantization law. To this purpose, the key point is the need to distinguish between the dynamics of the diagonal elements of 
TABLE 4: Example of average bit rates in the presence of alien crosstalk, in comparison with the nonvectored system and the vectored system without alien.

\begin{tabular}{lcccc}
\hline $\begin{array}{l}\text { Line length } \\
(\mathrm{km})\end{array}$ & $\begin{array}{c}\text { Vectored with } \\
\text { alien (Mbps) }\end{array}$ & $\begin{array}{c}\text { Nonvectored with } \\
\text { alien (Mbps) }\end{array}$ & $\begin{array}{c}\text { Vectored } \\
\text { without alien (Mbps) }\end{array}$ & $T_{1}^{n}$ \\
\hline 0.3 & 86.65 & 78.14 & 141.25 & $38.65 \%$ \\
0.6 & 69.50 & 65.06 & 94.19 & $26.22 \%$ \\
0.9 & 49.60 & 47.32 & 58.46 & $15.16 \%$ \\
1.2 & 34.81 & 33.38 & 37.67 & $7.38 \%$ \\
\hline
\end{tabular}

$\mathbf{P}_{k}$, that are close to 1 , and that of the off-diagonal elements, that are much smaller than 1 (because of the RWDD property). So, we propose to adapt the midtread quantization law to such dynamics, by assuming different quantization thresholds for the two classes of data. In practice, the $2^{v}$ quantization levels are distributed between $-T_{h 1}$ and $T_{h 1}$ for the diagonal elements, and between $-T_{h 2}$ and $T_{h 2}$ for the offdiagonal elements.

The assumption of $T_{h 1}$ equal to 1 seems a natural choice. On the contrary, the choice of $T_{h 2}$ should take into account the dynamics of the off-diagonal elements. Figure 6 shows an example of maximum and average values of $\left|P_{k}^{i j}\right|$, with $i \neq j$, for $L=8$ and line lengths $d=0.3 \mathrm{~km}$ and $d=1.2 \mathrm{~km}$, respectively. Looking at the average value, the assumption of $T_{h 2}=0.05$ is a reasonable choice, particularly for the shortest line lengths that are more frequent in practice. So, we propose to adopt two uniform quantization laws, but with different clipping, namely: $[-1,+1]$ for the diagonal elements and $[-0.05,+0.05]$ for the off-diagonal elements.

The proposed technique is indeed able to reduce the number of quantization bits, as shown in the next section. It should be noted that the implementation of this quantization scheme does not require any additional processing, but only a selective management of the elements of the precoding matrix.

6.2. Numerical Results: Performance in the Presence of Quantization Errors. Let us consider a scenario with $L=8$ lines having the same length. We simulate four different values of the line length, namely, $d=0.3 \mathrm{~km}, d=0.6 \mathrm{~km}, d=$ $0.9 \mathrm{~km}, d=1.2 \mathrm{~km}$. Tables 5 and 6 show the values of $\left\langle L^{n}\right\rangle /\left\langle C^{n}\right\rangle \cdot 100$ as obtained by the model in [20] and by the midtread quantization law. The difference between the two groups of results is evident for small $v$, while it becomes smaller and smaller for larger $v$. Both Tables 5 and 6 confirm that, wishing to have a rate loss below $2 \%$ for line length $\geq 0.3 \mathrm{~km}, v \geq 14$ bits is required. Though this value could be implemented on the basis of the current technology, it seems exaggeratedly high.

Let us investigate if the "double-threshold" quantization rule, proposed in the previous subsection, allows to reduce the number of quantization bits. So, for the same scenario above, let us assume a midtread quantization law with $T_{h 1}=1$ and $T_{h 2}=0.05$. The corresponding normalized losses are shown in Table 7, as functions of the number of quantization bits. In comparison with Tables 5 and 6, there is an improvement for any value of $v$. In particular, the target
TABLE 5: $\left\langle L^{n}\right\rangle /\left\langle C^{n}\right\rangle \cdot 100$ with uniform generation of the quantization errors.

\begin{tabular}{lccccc}
\hline $\begin{array}{l}\text { Line length } \\
(\mathrm{km})\end{array}$ & $v=6$ & $v=8$ & $v=10$ & $v=12$ & $v=14$ \\
\hline 0.3 & 80.87 & 53.37 & 28.06 & 9.86 & 1.91 \\
0.6 & 60.91 & 34.14 & 14.90 & 4.25 & 0.60 \\
0.9 & 56.38 & 30.00 & 12.20 & 3.23 & 0.39 \\
1.2 & 57.59 & 29.26 & 11.44 & 2.90 & 0.37 \\
\hline
\end{tabular}

TABLE 6: $\left\langle L^{n}\right\rangle /\left\langle C^{n}\right\rangle \cdot 100$ with midtread quantization law.

\begin{tabular}{lccccc}
\hline $\begin{array}{l}\text { Line length } \\
(\mathrm{km})\end{array}$ & $v=6$ & $v=8$ & $v=10$ & $v=12$ & $v=14$ \\
\hline 0.3 & 54.32 & 43.98 & 25.86 & 9.59 & 1.91 \\
0.6 & 31.88 & 24.19 & 12.82 & 4.08 & 0.63 \\
0.9 & 22.61 & 18.10 & 9.71 & 2.99 & 0.40 \\
1.2 & 18.28 & 15.19 & 8.51 & 2.65 & 0.38 \\
\hline
\end{tabular}

TABLE 7: $\left\langle L^{n}\right\rangle /\left\langle C^{n}\right\rangle \cdot 100$ with midtread quantization law adopting different thresholds.

\begin{tabular}{lccccc}
\hline $\begin{array}{l}\text { Line length } \\
(\mathrm{km})\end{array}$ & $v=6$ & $v=8$ & $v=10$ & $v=12$ & $v=14$ \\
\hline 0.3 & 24.01 & 8.69 & 1.97 & 0.80 & 0.68 \\
0.6 & 11.56 & 3.44 & 0.68 & 0.27 & 0.25 \\
0.9 & 8.63 & 2.39 & 0.29 & 0.05 & 0.04 \\
1.2 & 7.53 & 2.07 & 0.28 & 0.03 & 0.02 \\
\hline
\end{tabular}

of capacity loss below $2 \%$ for $d \geq 0.3 \mathrm{~km}$ can be achieved by using only $v=10$ bits, with a significant saving with respect to the case with equal thresholds.

\section{Conclusions}

With the development of accurate mathematical models of the FEXT in VDSL systems, it becomes feasible to find simple analytical formulas that describe the impact of some key practical impairment parameters on the achievable bit rate and the other performance figures. In this paper, we have first focused on the impact of FEXT coefficient estimation errors, by deriving formulas holding for absolute errors induced by the estimating algorithms and relative errors due to channel changes. The analysis also provides a simple evaluation of the maximum length where estimation errors reduce the coordinate system performance to that 


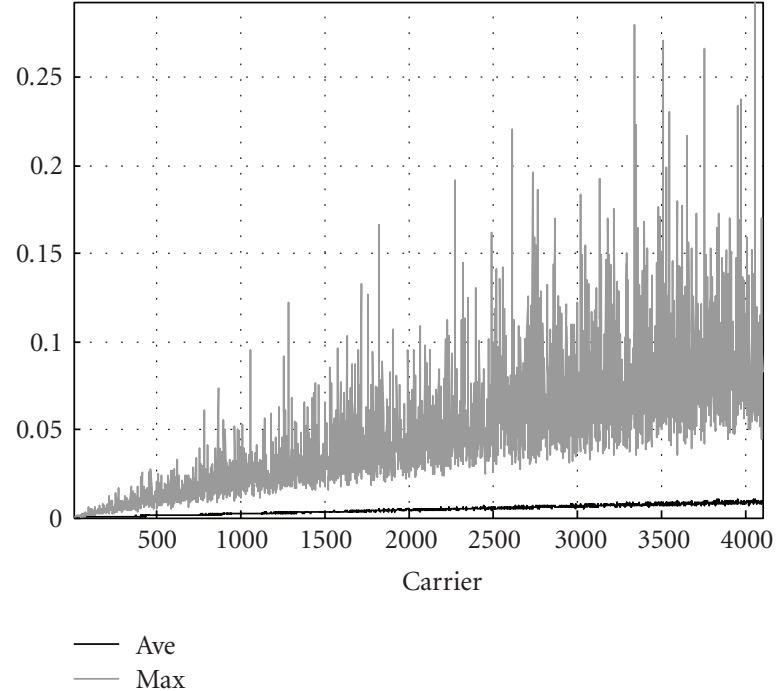

(a)

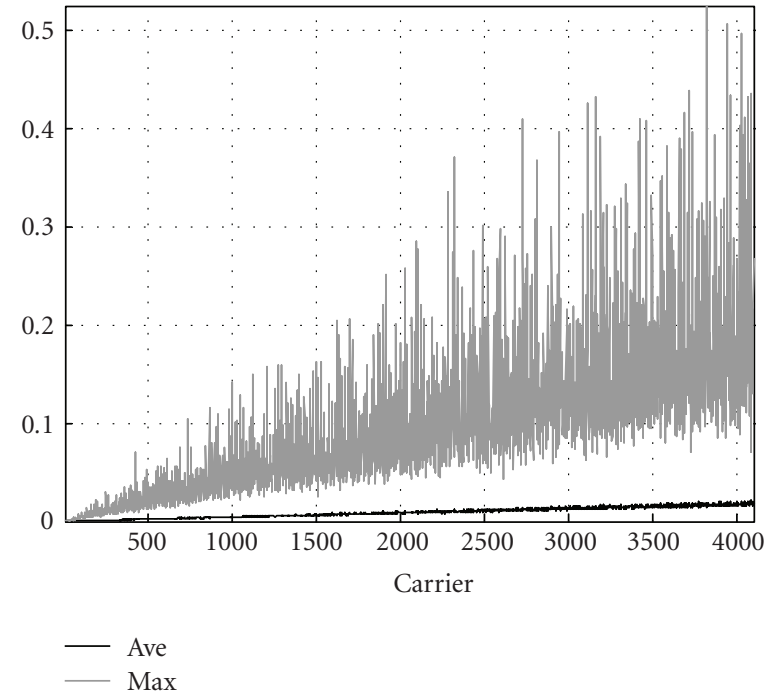

(b)

FIGURE 6: Simulated dynamics for the modulus of the off-diagonal elements of the precoding matrix: (a) $d=0.3 \mathrm{~km}$, (b) $d=1.2 \mathrm{~km}$.

of the noncoordinate one. Then, out-of-domain crosstalk sources have been considered: numerical results obtained from the presented formulas show that their impact can be very relevant and may represent a strong drawback for coordinate systems. Finally, the effect of quantization errors in the precoding matrix representation has been analyzed, by showing the advantage of a midtread quantization law using different thresholds. Among the most relevant conclusions of our study, we mention the limited dispersion of the bit rates around the estimated mean value, which makes the latter a reliable measure of the system performance. The simple analytical treatment presented in this paper provides useful preliminary information that can guide the system design and point out its potentialities, before resorting to practical measurements in the field.

\section{Appendices}

\section{A. Proof of the Approximation $\operatorname{diag}\left(\mathbf{E}_{k} \cdot \hat{\mathbf{H}}_{k}^{-1}\right) \approx 0$}

Let us consider the following singular value decomposition (SVD) for the estimated channel matrix $\hat{\mathbf{H}}_{k}$

$$
\hat{\mathbf{H}}_{k}=\mathbf{U}_{k} \cdot \boldsymbol{\Lambda}_{k} \cdot \mathbf{V}_{k}^{* T},
$$

where $T$ and $*$ denote the transpose and conjugate operations, respectively. In (A.1), $\mathbf{U}_{k}$ and $\mathbf{V}_{k}$ are orthogonal matrices containing the left-singular and right-singular vectors, while $\boldsymbol{\Lambda}_{k}$ is a diagonal matrix containing the singular values $\lambda_{k}^{i}, i=1 \ldots L$.

Similarly to the actual channel matrix $\mathbf{H}_{k}$, the estimated channel matrix $\hat{\mathbf{H}}_{k}$ is RWDD; this permits to approximate (A.1) as follows:

$$
\hat{\mathbf{H}}_{k} \approx \boldsymbol{\Lambda}_{k} \cdot \mathbf{V}_{k}^{* T}
$$

that also implies

$$
\hat{\mathbf{H}}_{k}^{* T} \approx \mathbf{V}_{k} \cdot \boldsymbol{\Lambda}_{k}^{* T}=\mathbf{V}_{k} \cdot \boldsymbol{\Lambda}_{k} .
$$

Combining (A.2) and (A.3), we have

$$
\hat{\mathbf{H}}_{k} \cdot \hat{\mathbf{H}}_{k}^{* T} \approx \boldsymbol{\Lambda}_{k} \cdot \mathbf{V}_{k}^{* T} \cdot \mathbf{V}_{k} \cdot \boldsymbol{\Lambda}_{k}=\boldsymbol{\Lambda}_{k}^{2} .
$$

Equation (A.4) implies

$$
\left(\lambda_{k}^{i}\right)^{2} \approx \sum_{m=1}^{L}\left|\hat{H}_{k}^{i m}\right|^{2} \approx\left|H_{k}^{i i}\right|^{2},
$$

where the assumption of null error for the channel matrix diagonal elements has also been taken into account. Moreover, we have

$$
\hat{\mathbf{H}}_{k}^{-1} \approx \hat{\mathbf{H}}_{k}^{* T} \cdot \boldsymbol{\Lambda}_{k}^{-2} \approx\left[\begin{array}{cccc}
\frac{H_{k}^{11 *}}{\left|H_{k}^{11}\right|^{2}} & \frac{\hat{H}_{k}^{21 *}}{\left|H_{k}^{22}\right|^{2}} & \cdots & \frac{\hat{H}_{k}^{L 1 *}}{\left|H_{k}^{L L}\right|^{2}} \\
\frac{\hat{H}_{k}^{12 *}}{\left|H_{k}^{11}\right|^{2}} & \frac{H_{k}^{22 *}}{\left|H_{k}^{22}\right|^{2}} & \cdots & \frac{\hat{H}_{k}^{L 2 *}}{\left|H_{k}^{L L}\right|^{2}} \\
\vdots & \vdots & \ddots & \vdots \\
\frac{\hat{H}_{k}^{1 L *}}{\left|H_{k}^{11}\right|^{2}} & \frac{\hat{H}_{k}^{2 L *}}{\left|H_{k}^{22}\right|^{2}} & \cdots & \frac{H_{k}^{L L *}}{\left|H_{k}^{L L}\right|^{2}}
\end{array}\right],
$$


having used the RWDD character of the channel matrix. Finally, multiplying (A.6) by (16), we obtain

$$
\mathbf{E}_{k} \cdot \hat{\mathbf{H}}_{k}^{-1} \approx\left[\begin{array}{cccc}
0 & \frac{\epsilon_{k}^{12}}{H_{k}^{22}} & \cdots & \frac{\epsilon_{k}^{1 L}}{H_{k}^{L L}} \\
\frac{\epsilon_{k}^{21}}{H_{k}^{11}} & 0 & \cdots & \frac{\epsilon_{k}^{2 L}}{H_{k}^{L L}} \\
\vdots & \vdots & \ddots & \vdots \\
\frac{\epsilon_{k}^{L 1}}{H_{k}^{11}} & \frac{\epsilon_{k}^{L 2}}{H_{k}^{22}} & \cdots & 0
\end{array}\right],
$$

which demonstrates that, in the order of approximation used in this paper, $\operatorname{diag}\left(\mathbf{E}_{k} \cdot \hat{\mathbf{H}}_{k}^{-1}\right) \approx \mathbf{0}$.

\section{B. Power of the Absolute Error in Case of Vector Approach for the in-Domain Crosstalk Estimation}

Following [4], let us suppose that all the elements of the $i$ th row $\mathbf{h}_{k}^{i}=\left[H_{k}^{i 1}, H_{k}^{i 2}, \ldots, H_{k}^{i L}\right]$ of the channel matrix, when $L$ lines are active, are simultaneously estimated by using an LS method. Let us define

$$
\begin{gathered}
\mathbf{X}_{k}(s)=\left[X_{k}^{1}(s), X_{k}^{2}(s), \ldots, X_{k}^{L}(s)\right]^{T}, \\
Y_{k}^{i}(s)=\sum_{j=1}^{L} H_{k}^{i j} X_{k}^{j}(s)+N_{k}^{i}(s), \\
\mathbf{Z}_{k}^{i}(S)=\sum_{s=1}^{S} Y_{k}^{i}(s)\left[\mathbf{X}_{k}(s)\right]^{* T}, \\
\mathbf{R}_{k}(S)=\sum_{s=1}^{S} \mathbf{X}_{k}(s)\left[\mathbf{X}_{k}(s)\right]^{* T},
\end{gathered}
$$

where $S$ is the number of training symbols used. The mean square error of the estimation is minimized by assuming

$$
\widehat{\mathbf{h}}_{k}^{i}=\mathbf{Z}_{k}^{i}(S)\left[\mathbf{R}_{k}(S)\right]^{-1} .
$$

It is well known [32] that the accuracy of the estimation is increased by adopting training sequences on different lines that are orthogonal across time and with equal power. A rather common choice is to use training sequences that are taken by the rows of a Walsh-Hadamard matrix. Let us suppose, w.l.o.g., that $L$ is a power of 2. When $S=x L$, with $x$ an integer $\geq 1$, matrix $\mathbf{R}_{k}(S)$ is diagonal, and its inversion is immediate. In the other cases, the recursive formula presented in [4] can be adopted.

The error is computed over the whole vector $\mathbf{h}_{k}^{i}$, resulting in an $L$-component row vector as follows:

$$
\epsilon_{k}^{i}(S)=\widehat{\mathbf{h}}_{k}^{i}-\mathbf{h}_{k}^{i}=\sum_{s=1}^{S} N_{k}^{i}(s)\left[\mathbf{X}_{k}(s)\right]^{* T}\left[\mathbf{R}_{k}(S)\right]^{-1} .
$$

Through simple calculations, the average value of the square modulus of $\epsilon_{k}^{i}(S)$ results in

$$
\left\langle\left|\epsilon_{k}^{i}(S)\right|^{2}\right\rangle=\sigma_{N}^{2} \cdot \operatorname{Tr}\left\{\left[\mathbf{R}_{k}(S)\right]^{-1}\right\},
$$

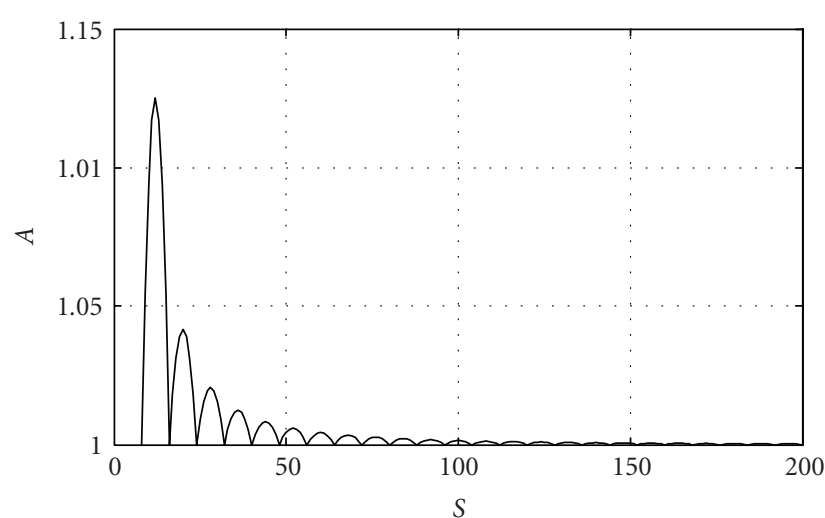

FIGURE 7: Normalized trace of $\left[\mathbf{R}_{k}(S)\right]^{-1}$ as a function of $S$.

where $\operatorname{Tr}(\mathbf{A})$ is the trace of matrix $\mathbf{A}$. In the case of $S=x L$, assuming that all lines transmit the same power $P_{k}$, we have

$$
\begin{aligned}
\left\langle\left|\epsilon_{k}^{i}(S)\right|^{2}\right\rangle & =\left\langle\left(\hat{H}_{k}^{i 1}-H_{k}^{i 1}\right)^{2}+\cdots+\left(\hat{H}_{k}^{i L}-H_{k}^{i L}\right)^{2}\right\rangle \\
& =\sigma_{N}^{2} \frac{L}{S \cdot P_{k}} .
\end{aligned}
$$

Sharing uniformly this power between the $L$ components of vector $\epsilon_{k}^{i}(S)$, expression (18) is attained (with $P_{k}^{j}=P_{k}$ ).

If $S \neq x L$, matrix $\left[\mathbf{R}_{k}(S)\right]^{-1}$ is no longer diagonal and the value of $\operatorname{Tr}\left\{\left[\mathbf{R}_{k}(S)\right]^{-1}\right\}$ depends on $S$. However, the following properties of $\left[\mathbf{R}_{k}(S)\right]^{-1}$ are general and can be easily checked: (i) the elements along the main diagonal are equal; (ii) the elements outside the main diagonal are greater than 1 . Property (ii), in particular, implies that the average square error is now greater than (B.5). But, as shown in Figure 7, that reports the normalized trace $A=S \cdot P_{k} \cdot \operatorname{Tr}\left\{\left[\mathbf{R}_{k}(S)\right]^{-1}\right\} / L$ for $L=8$ and $8 \leq S \leq 200$, the dependence on $S$ is weak: the normalized trace tends rapidly to 1 , and this implies that (B.5) can be applied, with very good approximation, for any practical value of $S$.

\section{Acknowledgments}

Part of this work has been funded by Telecom Italia S.p.A. The authors wish to thank Marco Burzio and Paola Cinato for helpful discussions, technical comments, and support. Part of these results has been presented at the Third International Conference on Communication Theory, Reliability, and Quality of Service (CTRQ 2010).

\section{References}

[1] R. Cendrillon, G. Ginis, E. van den Bogaert, and M. Moonen, "A near-optimal linear crosstalk precoder for downstream VDSL," IEEE Transactions on Communications, vol. 55, no. 5, pp. 860-863, 2007.

[2] G. Ginis and J. M. Cioffi, "Vectored transmission for digital subscriber line systems," IEEE Journal on Selected Areas in Communications, vol. 20, no. 5, pp. 1085-1104, 2002. 
[3] A. Leshem and L. Youming, "A low complexity linear precoding technique for next generation VDSL downstream transmission over copper," IEEE Transactions on Signal Processing, vol. 55, no. 11, pp. 5527-5534, 2007.

[4] P. Turcza and T. Twardowski, "RLS based MIMO channel identification for FEXT compensation in vectored $\mathrm{xDSL}$ system," in Proceedings of the European Conference on Circuit Theory and Design, pp. 251-254, Cork, Ireland, AugustSeptember 2005.

[5] C. Zeng, C. Aldana, A. A. Salvekar, and J. M. Cioffi, "Crosstalk identification in xDSL systems," IEEE Journal on Selected Areas in Communications, vol. 19, no. 8, pp. 1488-1496, 2001.

[6] G. Ysebaert, K. Vanbleu, G. Cuypers, and M. Moonen, "Split SR-RLS for the joint initialization of the per-tone equalizers and per-tone echo cancelers in DMT-based receivers," EURASIP Journal on Applied Signal Processing, vol. 2004, no. 10, pp. 1433-1445, 2004.

[7] P. Whiting, A. Ashikhmin, G. Kramer et al., "DSL crosstalk coefficient acquisition using SNR feedback," in IEEE Global Telecommunications Conference (GLOBECOM '08), pp. 3428 3432, New Orleans, La, USA, November-December 2008.

[8] J. Louveaux and A. J. van der Veen, "Error sign feedback as an alternative to pilots for the tracking of FEXT transfer functions in downstream VDSL," EURASIP Journal on Applied Signal Processing, vol. 2006, Article ID 94105, 14 pages, 2006.

[9] M. L. Gujrathi, J. Homer, I. V. L. Clarkson, R. Cendrillon, and M. Moonen, "A detection guided normalized least-meansquares adaptive partial crosstalk canceller for multi-user DSL environments," IEEE Signal Processing Letters, vol. 16, no. 6, pp. 489-492, 2009.

[10] M. Glavin and E. Jones, "Equalization of digital subscriber lines under dynamic channel conditions," Signal Processing, vol. 84, no. 5, pp. 853-864, 2004.

[11] R. Cendrillon, F. Liming, J. Chou, G. Long, and D. Wei, "Challenges and solutions in vectored DSL," in Proceedings of the International Conference on Access Networks, Hong Kong, November 2009.

[12] E. Karipidis, N. Sidiropoulos, A. Leshem, and L. Youming, "Experimental evaluation of capacity statistics for short VDSL loops," IEEE Transactions on Communications, vol. 53, no. 7, pp. 1119-1122, 2005.

[13] P. Whiting, A. Ashikhmin, S. Borst et al., "Performance results for digital subscriber line precoders," Bell Labs Technical Journal, vol. 13, no. 1, pp. 147-162, 2008.

[14] N. Lindqvist, F. Lindqvist, M. Monteiro, B. Dortschy, E. Pelaes, and A. Klautau, "Impact of crosstalk channel estimation on the DSM performance for DSL networks," EURASIP Journal on Advances in Signal Processing, vol. 2010, Article ID 935076, 11 pages, 2010.

[15] ITU-T Recommendation G.996.1, "Test procedures for digital subscriber line (DSL) transceivers," February 2006.

[16] G. Zimmermann, "On the importance of crosstalk from mixed sources,” T1E1.4/97-181, May 1997.

[17] "Spectrum Management for Loop Transmission Systems," Committee T1 Standard T1.417-2001, January 2001.

[18] M. Sorbara, P. Duvaut, F. Shmulyian, S. Singh, and A. Mahadevan, "Construction of a DSL-MIMO channel model for evaluation of FEXT cancellation systems in VDSL2," in IEEE Sarnoff Symposium (SARNOFF '07), pp. 1-6, Princeton, NJ, USA, April-May 2007.

[19] NIPP-NAI Subcommittee, "Multiple-Input Multiple-Output Crosstalk Channel Model," Proposed Draft Technical Report 2009-014R3, Indian Wells, Calif, USA, April 2009.
[20] E. Sayag, A. Leshem, and N. D. Sidiropoulos, "Finite word length effects on transmission rate in zero forcing linear precoding for multichannel DSL," IEEE Transactions on Signal Processing, vol. 57, no. 4, pp. 1469-1482, 2009.

[21] ITU-T Recommendation G.993.2, Amendment 1, "VTU-R limit PSD masks for band plan 998 (and its extensions)," Table B.6, Profile 998E17-M2x-NUS0, April 2007.

[22] W. Yu, "Multiuser water-filling in the presence of crosstalk," in Proceedings of the Information Theory and Applications Workshop (ITA '07), pp. 414-420, San Diego, Calif, USA, January-February 2007.

[23] J. Musson, "Maximum likelihood estimation of the primary parameters of twisted pair cables," ESTSI/STC TM6 981t08a1, Madrid, Spain, January 1998.

[24] A. Safak, "Statistical analysis of the power sum of multiple correlated log-normal components," IEEE Transactions on Vehicular Technology, vol. 42, no. 1, pp. 58-61, 1993.

[25] P. Cardieri and T. S. Rappaport, "Statistics of the sum of lognormal variables in wireless communications," in Proceedings of the 51 st IEEE Vehicular Technology Conference (VTC'00), pp. 1823-1827, Tokyo, Japan, May 2000.

[26] S. C. Schwartz and Y. S. Yeh, "On the distribution function and moments of power sums with log-normal components," The Bell System Technical Journal, vol. 61, no. 7, pp. 1441-1462, 1982.

[27] S. M. Kay, Fundamentals of Statistical Signal Processing, Volume 1: Estimation Theory, Prentice Hall, Englewood Cliffs, NJ, USA, 1993.

[28] ITU-T Draft Standard G.vector, "Self-FEXT cancellation (vectoring) for use with VDSL2 transceivers," Temporary Document 09Xc-R18R3, 2009.

[29] S. Galli and K. J. Kerpez, "Methods of summing crosstalk from mixed sources - part I: theoretical analysis," IEEE Transactions on Communications, vol. 50, no. 3, pp. 453-461, 2002.

[30] G. Ginis and C.-N. Peng, "Alien crosstalk cancellation for multipair digital subscriber line systems," EURASIP Journal on Applied Signal Processing, vol. 2006, Article ID 16828, 12 pages, 2006.

[31] R. Cendrillon, "Low-complexity out-of-domain crosstalk cancellation," private communication, 2009.

[32] B. Hassibi and B. M. Hochwald, "How much training is needed in multiple-antenna wireless links?" IEEE Transactions on Information Theory, vol. 49, no. 4, pp. 951-963, 2003. 